\title{
Computer assisted analysis of fluorescein videoangiograms
}

\author{
Paul A R Meyer, Fred W Fitzke
}

\section{Abstract}

The efficient analysis of fluorescein videoangiograms requires immediate access to any frame in a video sequence and complete control over the time base during replay. It should also be possible to compare any two frames by rapid subtraction or to subtract any phase of the study from the remaining sequence. A program that performs all these tasks has been written for a low priced desk-top microcomputer. This should facilitate the clinical introduction of fluorescein videoangiography.

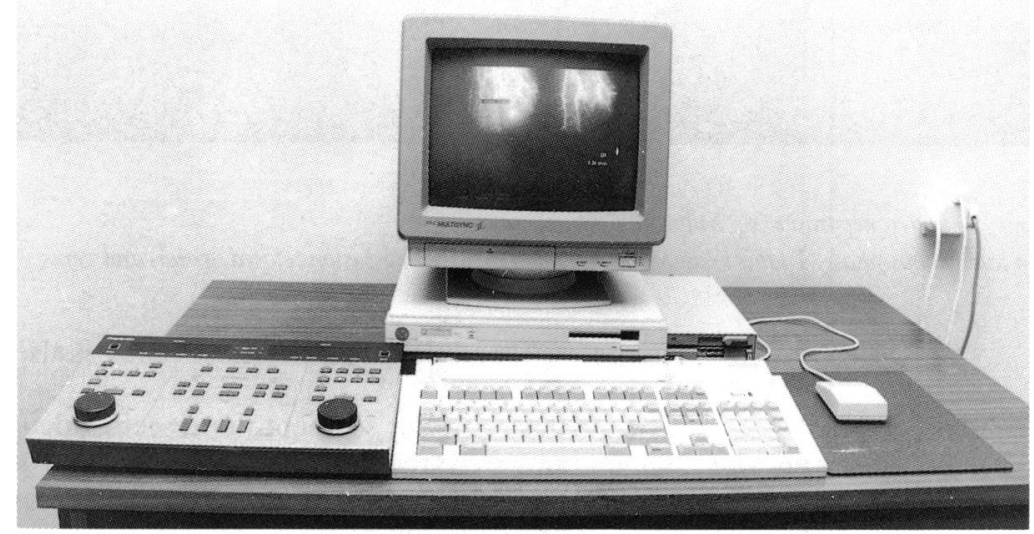

Figure 1: The Archimedes computer with mouse (right) and video controller (left). Images are presented in quarter screen format.
Fluorescein videoangiography, during which fields are captured every 0.02 second, records the dynamics of vascular perfusion as well as its anatomy. ${ }^{1+}$ In the episcleral circulation this has clarified the distinction between arteries and veins. ${ }^{+}$It has also revealed interarterial communications and confirmed that anterior ciliary arteries may carry blood away from the limbus. ${ }^{+5}$ However, clinical fundus and anterior segment videoangiography have never become widely established.

The spatial resolution of the best video cameras is lower than that achieved by photography, and this is further reduced by all but the most expensive recording systems. However, the major hindrance to clinical videoangiography has been the difficulty of interpreting video images. When an ophthalmologist reads a photographic angiogram he examines each part of the sequence at a different rate, sometimes repeatedly roving forwards and backwards in time. This is a function that most video recorders perform very poorly.

The system that is presented here uses a fast desk top microcomputer to give the observer instant access to any location in an angiographic sequence and complete control of the rate at which images are displayed. Any two fields may be compared by the use of subtraction.
Addenbrooke's Hospital, Hills Road, Cambridge P A R Meyer

\section{Institute of}

Ophthalmology, Judd Street, London WC1 F W Fitzke

Correspondence to: Dr P A R Meyer, Department of Ophthalmology Addenbrooke's Hospital, Hills Road, Cambridge CB2 2QQ. Accepted for publication 30 November 1989

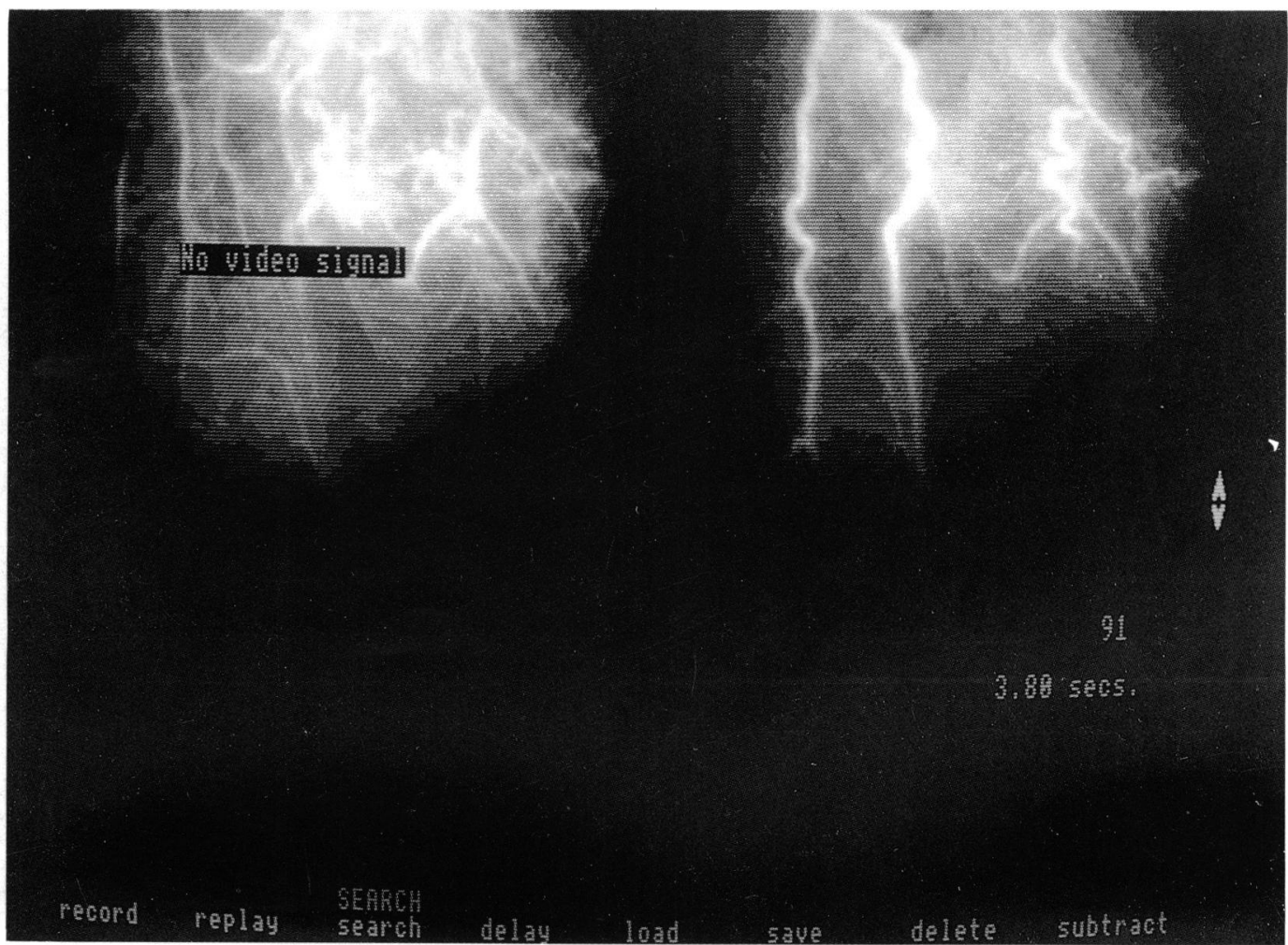

Figure 2: Standard screen format during 'search' routine. Left: most recent line image. Right: selected RAM image. Menu (bottom line) is always present and accessible by mouse. 


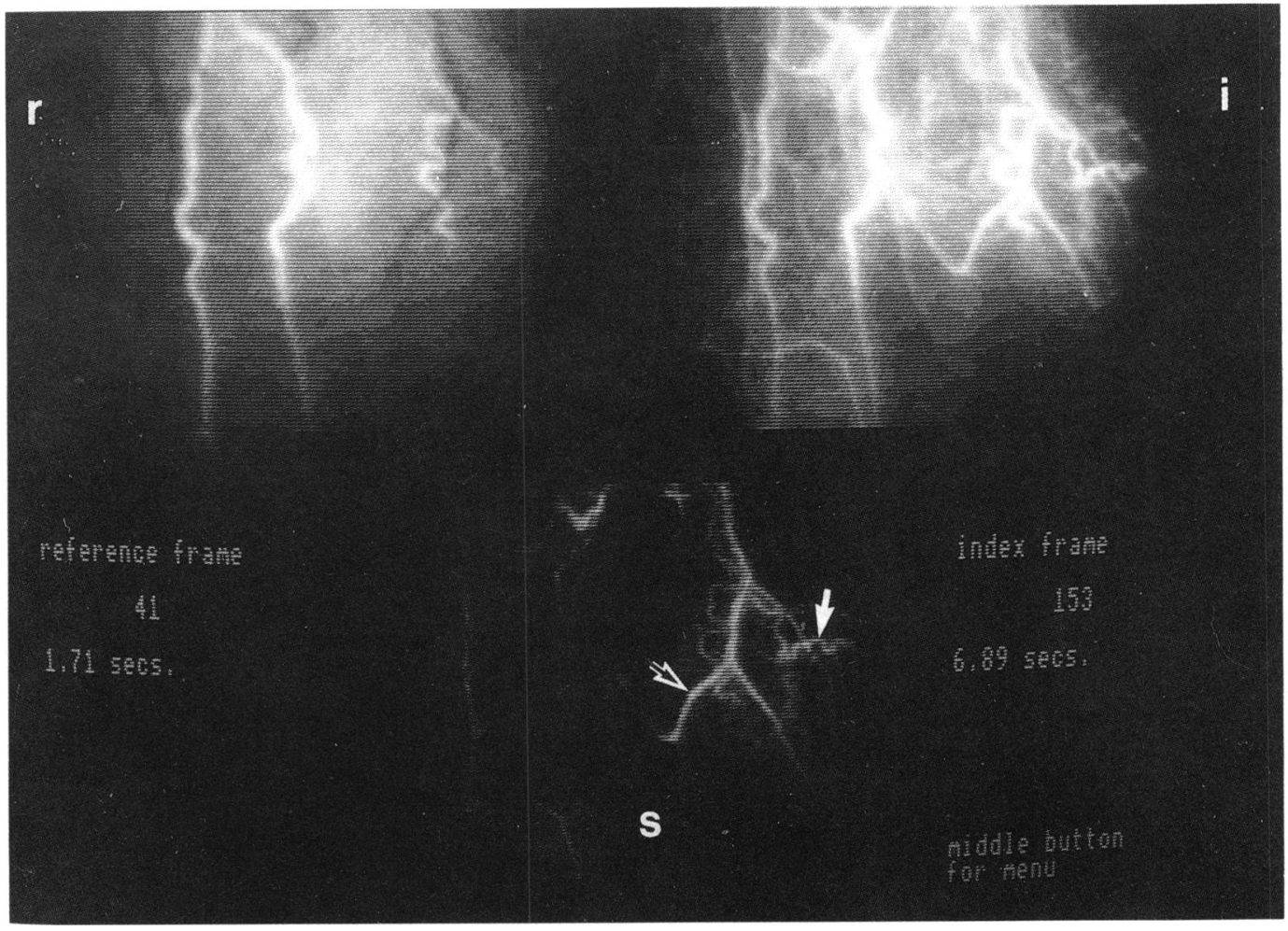

Figure $3 A$

Figure 3: Subtraction: Index frame (i). Reference frame (r). Subtracted image (s).

A: Arterial phase subtracted from late venous phase. In this example arteries with delayed perfusion (closed arrow) and veins (open arrow) are demonstrated.

\section{Methods and results}

HARDWARE

The image analysis system is based on the Archimedes 440 computer (Acorn, Cambridge). This is equipped with 4-megabytes RAM and a 20-megabyte hard disc. A video digitiser and frame store 'podule' (Watford Electronics) is also required (Fig 1).

Low-dose anterior segment fluorescein videoangiograms were recorded on to U-matic videotape by an image intensified Nuvicon camera, as described previously. ${ }^{4}$

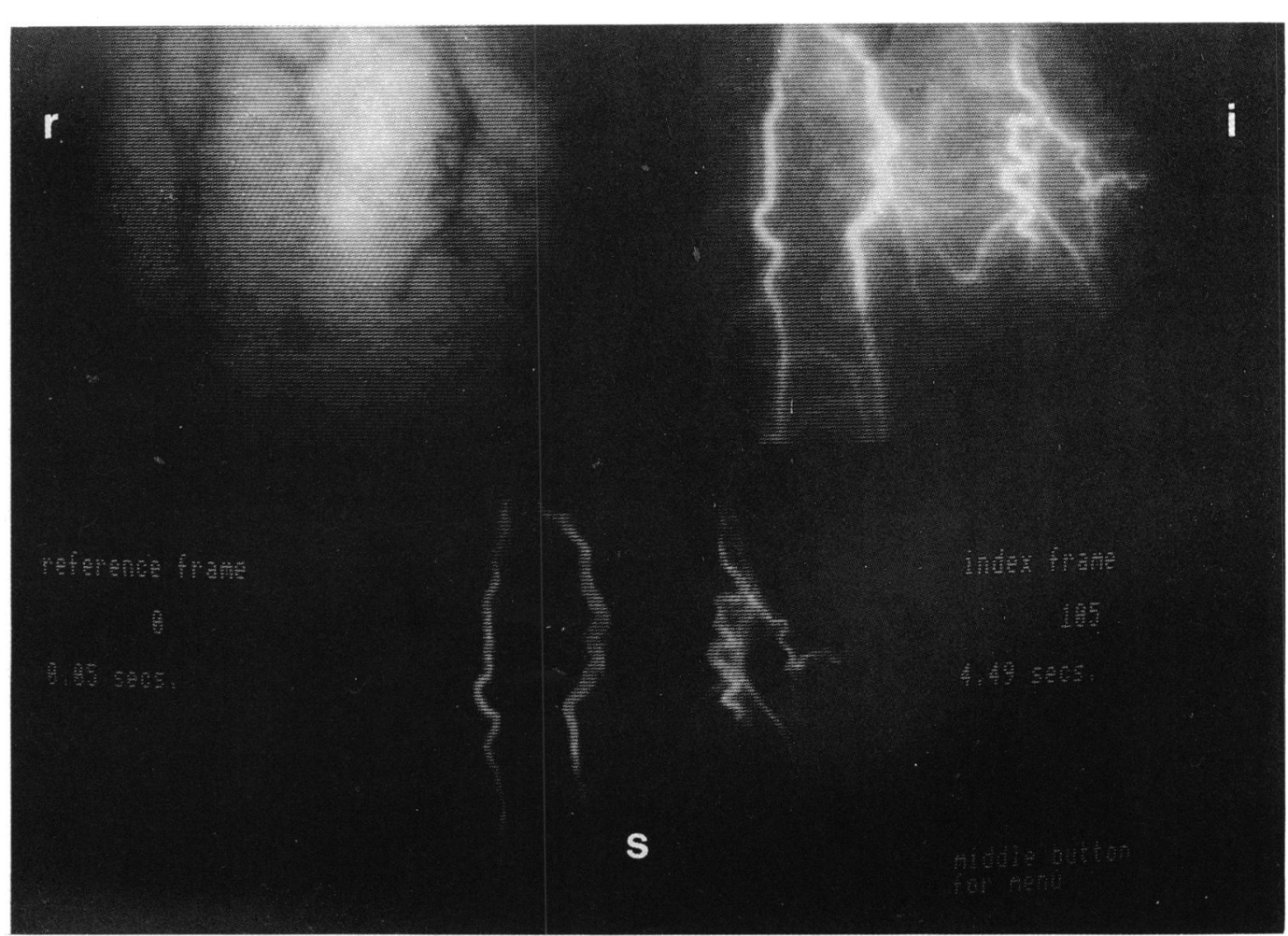

Figure $3 B$

B: Background (auto- and pseudofluorescence) subtracted from arterial phase to enhance contrast. 


\section{SOFTWARE}

The program is written in structured Basic and machine code. It is accessed entirely by means of the mouse, and a 'menu' remains continually on screen (Fig 2).

A video sequence is fed from a camera or videotape into the video digitiser, and the rate of transfer of frames to RAM is selected by the operator (maximum 12.5 frames/s). Up to 180 frames may be stored as $125 \times 125$ pixel images with 6 bit resolution (64 grey levels).

Images are displayed at 4 bit (16 grey level) resolution as quarter screens on a multisync monitor (NEC). This allows room to present frames from different memory locations simultaneously - usually the 'line' image in the digitiser memory and the image from the RAM location that is currently being examined (Figs 1, 2).

The video sequence (or any part of it) may be played forwards or backwards repeatedly. The transition from one frame to another is seamless.

Sequences can also be 'searched'. During this procedure the position of the mouse determines the frame that is currently displayed. By moving the mouse towards or away from him, the operator has complete control of both the moment in a sequence and the rate of display (Fig 2).

By means of a machine code subroutine two images may be subtracted in less than $0 \cdot 1$ second, allowing almost instant comparison (Fig $3 \mathrm{~A})$ or the erasure of background fluorescence (Fig 3B). Superimposition of frames is achieved by flickering between them while one is held static and the other is moved horizontally or vertically by the mouse. Alternatively, one frame (representing, for example, choroidal fluorescence or scleral autofluorescence) may be rapidly subtracted from the entire sequence. The computer memory is then updated so that the processed sequence may be replayed or searched just as were the raw data.

The program also includes facilities for measuring distances between points (or the length of a drawn line) in successive frames, which is helpful for assessing flow velocities or the spread of capillary perfusion.

Any raw or subtracted sequence may be stored on hard disc for future retrieval.

\section{Discussion}

With the help of a powerful, fast desk top microcomputer an ophthalmologist may be given access to an entire video sequence with a directness hitherto impossible. Frames from different moments in the sequence can be rapidly compared by subtraction, and the contrast of whole sequences may be similarly enhanced.

The system has now been used for the analysis of arterial and venous blood flow direction and velocity in 20 experimental episcleral video- angiograms from normal subjects. It proved to be both more consistent and approximately 10 times faster than a previous manual method involving direct measurements from a monitor.

When a videoangiogram is displayed seamlessly at high speed, the moment of peak capillary fluorescence during first pass of the dye is clearly demonstrated. This is a time point that is independent of illumination or camera sensitivity threshold. It has been used to quantify regional filling delays within an angiographic field, particularly during the interpretation of experimental iris fluorescein videoangiograms.

Clinical angiograms from patients with scleritis and corneal neovascularisation induced by wearing a contact lens have also been successfully analysed. Although designed for fluorescein angiography, this method of image analysis is equally applicable to radiology (for example, barium swallow or cardiac catheterisation) and laboratory microscopy (for example, the observation of cultured cells). The system that is described here requires only one monitor, is very reasonably priced, and has adequate resolution for most clinical purposes. For short video sequences a recorder is not required, and time lapse video recording is also possible.

However, the presentation of all images by the computer's own display restricts the grey scale to 16 levels, and the $125 \times 125$ pixel format lowers spatial resolution. The program is therefore being adapted for use with a new video digitiser (Hawk V10; Wildvision) that is capable of capturing $256 \times 256$ pixel images at 8 bit resolution and down-loading them to RAM at 0.2 second intervals. They are displayed with 8 bit resolution on a separate monitor.

Static images are inadequate for examining dynamic events such as blood flow. Although video may result in lower spatial resolution than photography, this is offset by the greatly enhanced temporal resolution. The provision of complete control over the time base of an angiogram offers the clinician an overview of vascular perfusion that has previously been impossible and should facilitate the clinical application of videoangiography.

The evolution of this image processing system, particularly the use of a mouse to control the presentation of a video sequence, owe much to discussions with Mike Hobart.

Paul A R Meyer is in receipt of a research fellowship from the Wellcome Trust. Fred W Fitzke gratefully acknowledges support from the Wellcome Trust and the National Retinitis Pigmentosa Foundation.

1 Marsh RJ, Ford SM. Cine photography and video recording of anterior segment fluorescein angiography. $\mathrm{Br} \mathcal{F}$ Ophthalmol 1978;62: 657-9.

2 Haining WM. Video funduscopy and fluoroscopy. Br $\mathcal{F} \mathrm{Oph}$ thalmol 1981; 65: 702-6.

3 Favilla I, Barry WR, Turner IJ. Video and digital fluorescein angiography. Aust N Z F Ophthalmol 1986; 14: 229-34.

4 Meyer PAR. Patterns of blood flow in episcleral vessels studied by low-dose fluorescein videoangiography. Eye 1988; 2: 533-46.

5 Meyer PAR. The circulation of the human limbus. Eye 1989; 3 : $121-7$ 